\title{
Qualité microbiologique des tranches de mangues (Mangifera indica L.) vendues à Dakar (Sénégal)
}

\author{
Moussa KASSE ${ }^{1,2,3}$, Mady CISSE${ }^{2}$, Aminata TOURE 2 , \\ Marie-Noëlle DUCAMP-COLLIN ${ }^{3}$ et Aliou GUISSE ${ }^{1,4^{*}}$ \\ ${ }^{I}$ Département de Biologie Végétale, Université Cheikh Anta Diop (UCAD), BP 5005, Dakar, Sénégal. \\ ${ }^{2}$ Ecole Supérieure Polytechnique (ESP), UCAD, BP 5085, Dakar - Fann, Sénégal. \\ ${ }^{3}$ Centre de coopération Internationale de Recherches Agronomiques pour le Développement (CIRAD), \\ Persyst, UMR 95 Qualisud, TA B-95/16, Avenue Agropolis, 34398 Montpellier cedex 5, France. \\ ${ }^{4}$ OHM Tessekere et UMI 3189, Sénégal. \\ *Auteur correspondant, E-mail: alguisse@orange.sn; Tel :00221 776389669.
}

\section{RESUME}

La mangue, principal fruit du Sénégal, est transformée en tranches et vendue en sachets dans les rues de Dakar. Ce produit est sensible aux altérations microbiennes faute de technologies de conservation adaptées au contexte local. Ce travail propose une évaluation de la qualité microbiologique du produit et de son impact sur la santé des consommateurs. Pour cette étude, 3 répétitions d'échantillons par vendeur ont été prélevées chez quatorze vendeurs de cinq quartiers (Médina, Fann, Habitats Loyers Modernes ou HLM, Grand Yoff et Guédiawaye). Ensuite, des analyses ont été menées pour la recherche de la flore mésophile aérobie totale, de coliformes, d'entérobactéries et d'Escherichia coli présomptifs. Les résultats montrent une forte pollution des échantillons en flore totale sauf pour l'échantillon V3 (vendeur 3) des HLM et une charge élevée en coliformes et en Escherichia coli (> $\left.10^{3} \mathrm{UFC} / \mathrm{g}\right)$. D'autres entérobactéries comme Enterobacter agglomerans, Enterobacter amnigenus, Serrati rubidaea et Klebsiella pneumoniae sont retrouvées respectivement dans les échantillons V2 de Médina, V1 de Fann, V1 et V2 Guédiawaye. Ces bactéries ne présentent pas de risques sur la santé publique mais rendent le produit impropre à la consommation. Cette étude nécessite une expansion aux autres quartiers pour mieux veiller sur la santé des consommateurs.

(C) 2014 International Formulae Group. All rights reserved.

Mots clés: Evaluation, qualité, microbiologie, mangues, santé des consommateurs, Dakar.

\section{INTRODUCTION}

Dans les pays du Sud, une proportion importante de fruits et légumes est actuellement perdue après la récolte, faute de technologies de conservation et de transformation adaptées aux contextes locaux (Reynes et Ducamp, 2008). Les pertes post récoltes sont chiffrées à environ $80 \%$ dans le monde pour la mangue (Kansci et al., 2003). Temple (2001) estime ces pertes à environ
$60 \%$ pour les mangues camerounaises, un taux relativement comparable aux pertes post récoltes de mangues au Sénégal (Mbodj, 2005). Quelle technologie adopter alors pour réduire ces pertes?

C'est la raison pour laquelle la transformation traditionnelle des fruits et légumes est devenue une pratique répandue dans les pays du Sud et qui, de nos jours, est 
entrain de prendre de l'ampleur en Afrique de l'ouest.

Les fruits et légumes contiennent des fibres, des minéraux, des vitamines, des polyphénols et des caroténoïdes, des éléments essentiels pour notre santé (James et Kuipers, 2003). Ces précieux constituants sont intacts dans les fruits fraîchement cueillis; mais les procédés agroalimentaires et les pratiques culinaires peuvent les détruire. A l'échelle de la planète, l'essentiel des fruits est transformé en boissons, en compotes, en confitures et en conserves (Cirad, 2009).

Plusieurs raisons justifient donc le recours à des technologies appropriées permettant de conserver la qualité initiale des produits. Il s'agit, entre autres des exigences sanitaires (Thuan, 2003; Kassé, 2009) et de l'apparition de nouvelles habitudes alimentaires dues à une urbanisation et à une généralisation du travail des femmes qui passent de moins en moins de temps à la préparation des repas (Buffet, 2003). Selon Artès (2004), les raisons peuvent être liées au grignotage, à la restauration hors foyer et à une tendance à vouloir une alimentation saine et diversifiée. La sensibilité des produits aux organismes pathogènes (Sandhya, 2010) et aux pertes nutritionnelles engendrées par les procédés agroalimentaires (Cirad, 2009; Cissé et al., 2011) sont en outre, deux raisons à ne pas négliger pour le maintien de la qualité des fruits. Par ailleurs, les consommateurs s'intéressent de plus en plus à de nouveaux produits élaborés, tant à l'internationale que localement. Ainsi, le marché des produits de quatrième $\quad\left(4^{\text {ème }}\right)$ gamme s'est considérablement développé en termes de quantité et de variété de produits disponibles pour les consommateurs (Artés, 2004). Un exemple classique des produits de $4^{\text {ème }}$ gamme est la salade en sachets, développée en Europe dans les années 80. Ces derniers sont définis comme étant des produits frais, crus, prêts à l'emploi ou «ready-to-use fresh product» (Varoquaux, 2002). Pour Buffet (2003), il s'agit de produits pelés, parés, découpés et pouvant être conditionnés sous atmosphère modifiée ou «fresh-cut products ». D'autres auteurs (Djioua, 2010; Djioua et al., 2010) considèrent tout simplement la quatrième gamme comme des produits frais crus ayant été faiblement transformés et conditionnés dans des emballages spécifiques puis conservés à une température comprise entre 4 et $6{ }^{\circ} \mathrm{C}$ qu'ils appellent en termes anglais « minimally processed products $»$.

$\mathrm{Au}$ Sénégal, la mangue, principale production fruitière avec plus de $60 \%$ (Mbodji, 2005), est confrontée à des problèmes de conservation, à l'état frais, dus à la mouche des fruits. C'est ainsi que, les détaillants de fruits au Sénégal ont adopté une pratique consistant à vendre les mangues en tranches conditionnées dans des sachets plastiques (Figure 1), à l'image des mangues en $4^{\text {ème }}$ gamme. Derrière ce bienfait technologique, notamment du point de vue de la valorisation du fruit, il se cache beaucoup de facteurs non contrôlés tels que l'innocuité de la matière première, l'hygiène dans la préparation, les paramètres physicochimiques et donc des défauts de qualité du produit fini pouvant impacter sur la santé des consommateurs.

L'objet de ce travail est d'évaluer la qualité microbiologique des tranches de mangues vendues en sachets dans les rues à Dakar afin de proposer une technologie adaptée au contexte local pour la conservation de ce produit. En effet, l'évaluation de la qualité microbiologique d'un produit alimentaire est tributaire d'un certain nombre d'indicateurs et de critères, liés à la qualité hygiénique et à la qualité commerciale de l'aliment. La qualité hygiénique (ou sécurité de l'aliment) caractérise le risque pour la santé des consommateurs et la qualité commerciale (ou salubrité de l'aliment) caractérise le risque d'altération au cours de la conservation de l'aliment. Pour ce faire, les germes naturellement recherchés sont: la flore 
mésophile aérobie totale (FMAT); les coliformes totaux et fécaux; Escherichia coli et les entérobactéries.

La FMAT permet d'avoir une indication sur le degré de contamination générale de l'aliment pour son acceptabilité à la consommation. Elle est aussi appelée flore d'altération de l'aliment.

Les coliformes totaux et fécaux donnent une idée sur les conditions d'hygiène lors de la fabrication et de la conservation du produit. Ce sont des indicateurs d'hygiène du procédé et de son environnement.

Escherichia coli est un coliforme qui témoigne d'une contamination fécale d'origine humaine et donc témoin de l'hygiène du transformateur. Il permet également d'avoir une indication sur la présence d'éventuelles souches entéropathogènes.

Les entérobactéries auxquels appartiennent les coliformes renferment également des souches pathogènes, dangereuses pour le consommateur. Ce sont des indicateurs de la sécurité de l'aliment (absence de danger). Il est donc important, s'ils sont présents dans certains échantillons, d'identifier les souches sur des galeries Api20E.

\section{MATERIEL ET METHODES}

\section{Matériel}

Cette étude a été réalisée sur des mangues en tranches durant la période de juin à juillet 2011. Cinq (5) quartiers (Fann, Médina, HLM ou Habitats Loyers Modernes, Grand Yoff et Guédiawaye) de la région de Dakar (Figure 2) ont été ciblés. Le choix des quartiers a été réalisé en fonction de la densité de la population, de leur proximité avec les marchés ou les lieux de regroupement des jeunes, principaux consommateurs du produit.

Dans chaque localité, les prélèvements ont été effectués auprès de 2 à 3 vendeurs (Tableau 1). Au niveau de chaque vendeur, 3 unités de vente de tranches de mangues ont été prélevées et acheminés dans une glacière au laboratoire de Microbiologie Appliquée et de Génie Industrielle (MAGI) de l'Ecole Supérieure Polytechnique (ESP) de Dakar pour une évaluation immédiate de leur qualité microbiologique.

Les germes recherchés dans ce travail sont: la flore mésophile aérobie totale; les coliformes totaux et fécaux; Escherichia coli et les entérobactéries. Ainsi, les milieux de culture plate count agar (PCA), Désoxycholate Citrate Lactose (DCL), rose gal BCIG (RG) et violet red bile dextrose (VRBD) ont été utilisés respectivement pour la culture et la recherche de la flore mésophile aérobie totale (FMAT), des coliformes fécaux et totaux, la culture et la recherche de Escherichia coli et pour le dénombrement des entérobactéries.

\section{Méthodes}

Les trois sachets (unités de vente) de tranches de mangues prélevés au niveau de chaque vendeur ont été mélangés pour constituer un échantillon global. Dix (10) grammes $(\mathrm{g})$ de cet échantillon ont été broyés dans un sachet stomacher et dilués dans $90 \mathrm{ml}$ d'eau peptonée salée (EPS). A partir de cette solution mère $\left(10^{-1}\right)$, trois autres dilutions décimales $\left(10^{-2}, 10^{-3}\right.$ et $\left.10^{-4}\right)$ ont été réalisées. Ensuite, $1 \mathrm{ml}$ de chacune des quatre dilutions a été ensemencé en profondeur dans des boîtes de Pétri en raison de deux répétitions de boîtes par dilution. Enfin, les boîtes inoculées et solidifiées ont été incubées à l'étuve à des températures de $30{ }^{\circ} \mathrm{C}$ pendant $72 \mathrm{~h}, 37{ }^{\circ} \mathrm{C}$ et $44^{\circ} \mathrm{C}$ pendant $24 \mathrm{~h}, 30^{\circ} \mathrm{C}$ pendant $24 \mathrm{~h}$ et à $37{ }^{\circ} \mathrm{C}$ pendant $24 \mathrm{~h}$ respectivement pour la FMAT, les coliformes totaux et fécaux, Escherichia coli et les entérobactéries. La lecture des résultats a été faite par dénombrement des colonies caractéristiques (par comptage) de chaque germe. Les résultats obtenus ont été exprimés en unité format colonies (UFC) par boîte; ces derniers sont 


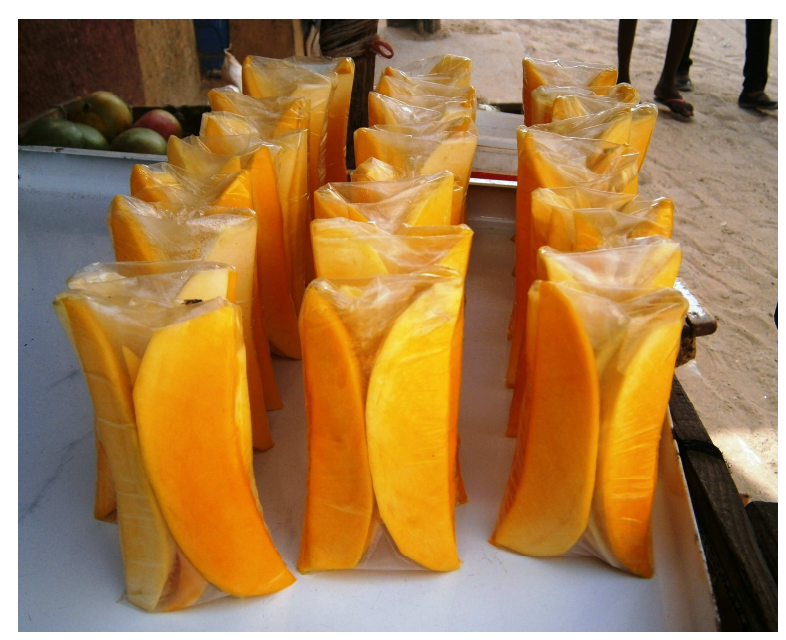

Figure 1 : Mangues en tranches vendues en sachets dans les rues à Dakar.

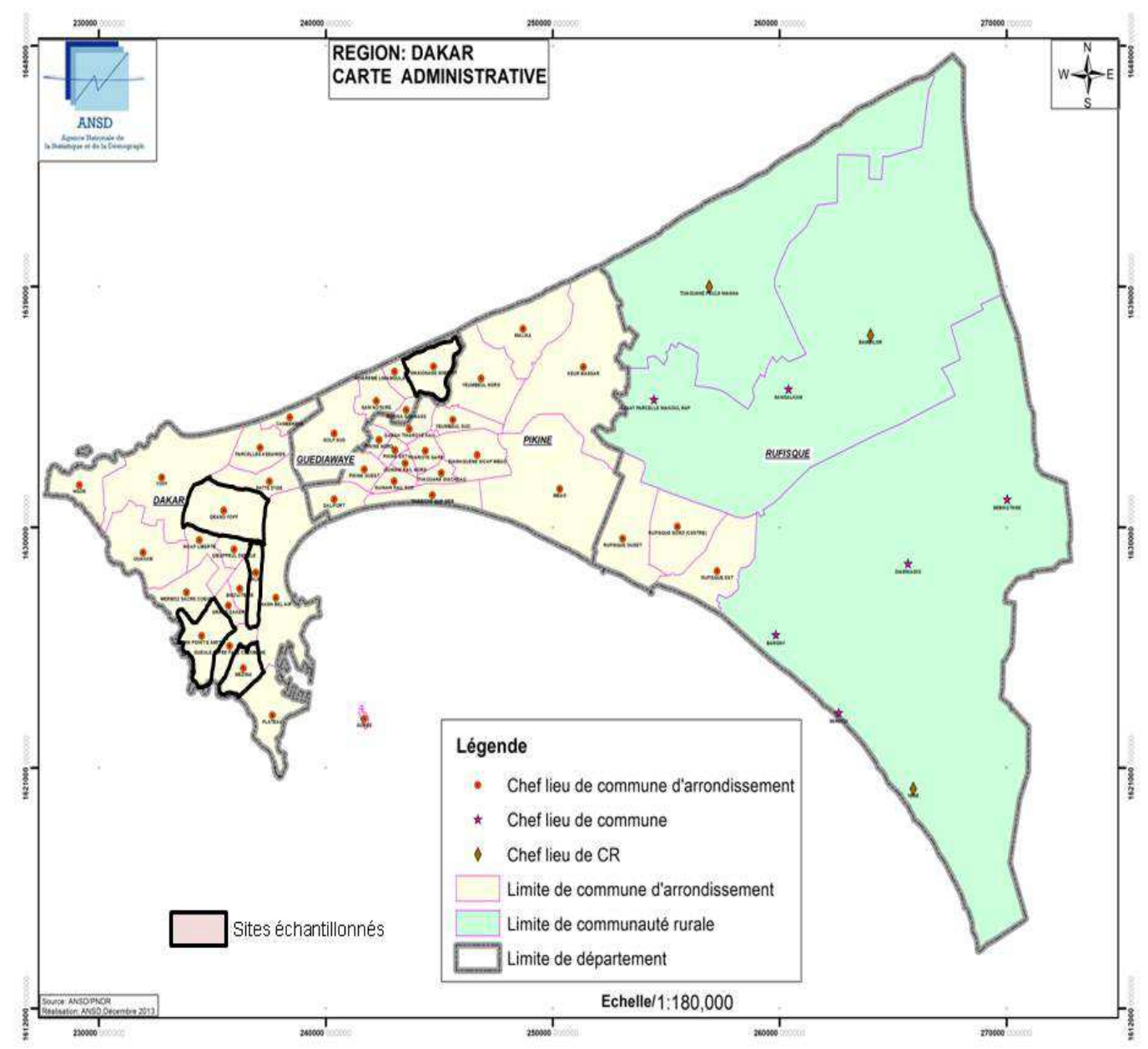

Figure 2 : Situation administrative des sites échantillonnés. (Source: ANSD, Février 2014). 
ensuite repris en UFC/g par l'application de la formule:

$$
N=\frac{\sum \text { Colonies }}{V m L^{*}\left(n 1+0,1^{*} n 2\right)^{*} D}
$$

où $\sum$ Colonies: somme des nombres de colonies bactériennes des boites considérées; $\mathrm{N}$ : nombre UFC par g de produit initial; VmL: Volume en $\mathrm{mL}$ ensemencé; $\mathrm{n} 1$ \& n2: nombre de boîtes interprétables choisies à la $1^{\text {ère }}$ et à la $2^{\text {ème }}$ dilution considérées et $\mathrm{D}$ : facteur de dilution de la $1^{\text {ère }}$ dilution considérée.

Des tests d'identification des entérobactéries sur Galerie Api20E ont été également réalisés sur les quatre (4) échantillons: V1 de Fann, V2 de Médina, V1 et V2 de Guédiawaye.

Les résultats ainsi obtenus ont été traités à l'aide du logiciel Microsoft Office Excel 2007 et les valeurs de N ont été calculées pour chaque flore étudiée en fonction des échantillons de chaque quartier, puis comparées à la référence normative des critères microbiologiques des aliments de l'homme (Règlement CE N ${ }^{\circ}$ 2073/2005). L'interprétation des résultats a été effectuée suivant le plan à 2 classes.

\section{RESULTATS}

Les résultats de l'analyse de la qualité microbiologique des tranches de mangues vendues en sachets dans les rues à Dakar (Tableau 2) montrent que la FMAT est élevée dans tous les échantillons sauf dans l'échantillon V3 des HLM. Elle est beaucoup plus importante dans les échantillons V3 de Fann $\left(1,49.10^{6} \mathrm{UFC} / \mathrm{g}\right)$ et V2 de Grand Yoff $\left(1,19.10^{6} \mathrm{UFC} / \mathrm{g}\right)$. Elle varie également d'un vendeur à l'autre au sein d'une même localité. En effet, les résultats des analyses pour la recherche de coliformes totaux et fécaux ont révélé une contamination très élevée des échantillons V2 de Fann et de Grand Yoff.

Cependant, les charges bactériennes en Escherichia coli et entérobactéries sont faibles pour la plupart des échantillons. Elles sont très élevées dans les échantillons V1 et V2 des HLM et V2 de Médina pour Escherichia coli; et dans les V1 et V2 de Grand Yoff et V3 des HLM en ce qui concerne les entérobactéries.

Des tests d'identification des entérobactéries sur galeries Api20E, réalisés sur les échantillons concernés, afin de rechercher la présence de germes pathogènes et d'évaluer le niveau de risque sur la santé des consommateurs, montrent la présence de Enterobacter agglomerans dans l'échantillon V2 de Médina, Enterobacter amnigenus dans le V1 de Fann, de Serrati rubidaea dans l'échantillon V1 de Guédiawaye et de Klebsiella pneumoniae dans celui V2 de Guédiawaye.

Tableau 1 : Répartition des Echantillons collectés par quartier.

\begin{tabular}{|c|c|c|}
\hline Quartiers & Echantillons & Rues / Adresses \\
\hline \multirow{3}{*}{ Fann } & V1 & Allée Claudel, UCAD \\
\hline & $\mathrm{V} 2$ & Avenue Cheikh Anta Diop \\
\hline & V3 & Couloir de la mort, UCAD \\
\hline \multirow{3}{*}{ Medina } & V1 & Rue 29 \\
\hline & $\mathrm{V} 2$ & Rue 6 \\
\hline & V3 & Rue 22 \\
\hline \multirow{3}{*}{ Hlm } & V1 & Hlm $3 x$ « garage guédiawaye » \\
\hline & $\mathrm{V} 2$ & Non précisée \\
\hline & V3 & Marché hlm 5 \\
\hline \multirow{3}{*}{ Grand yoff } & V1 & Marché grand Yoff \\
\hline & $\mathrm{V} 2$ & Rue, 359 \\
\hline & V3 & Rue, 353 \\
\hline \multirow{2}{*}{ Guediawaye } & V1 & Daroukhane, Terminus bus Dakar Dem Dikk \\
\hline & $\mathrm{V} 2$ & Marché Bou Bess \\
\hline
\end{tabular}


M. KASSE et al. / Int. J. Biol. Chem. Sci. 8(4): 1611-1619, 2014

Tableau 2 : Résultats des analyses microbiologiques des tranches de mangues vendues en sachet dans les rues à Dakar.

\begin{tabular}{|c|c|c|c|c|c|c|}
\hline \multirow[b]{2}{*}{ Flore recherchée } & \multirow[b]{2}{*}{ Echantillons } & \multicolumn{5}{|c|}{$\mathbf{N}($ UFC/g) } \\
\hline & & Fann & Medina & HIm & Grand Yoff & Guediawaye \\
\hline \multirow{2}{*}{ FMAT } & V1 & $1,47.10^{5}$ & $1,82.10^{3}$ & $1,08.10^{5}$ & $1,73.10^{4}$ & $2,09.10^{4}$ \\
\hline & V3 & $1,49.10^{6}$ & $1,82 \cdot 10^{3}$ & 0 & $3,18.10^{4}$ & - \\
\hline \multirow[b]{2}{*}{ Coliformes totaux } & V1 & $2,73.10^{3}$ & 0 & $7,27.10^{3}$ & $2,73.10^{3}$ & 0 \\
\hline & $\mathrm{V} 2$ & $3,55.10^{4}$ & 0 & 0 & $2,36.10^{5}$ & 0 \\
\hline \multirow[t]{2}{*}{ Coliformes fécaux } & $\mathrm{V} 2$ & $1,27.10^{4}$ & $1,82.10^{3}$ & 0 & $9,41.10^{4}$ & 0 \\
\hline & V3 & 0 & 0 & 0 & 0 & - \\
\hline \multirow{3}{*}{ Escherichia coli } & V1 & 0 & 0 & $9,09.10^{3}$ & 0 & $9,09.10^{2}$ \\
\hline & $\mathrm{V} 2$ & 0 & $2,73.10^{3}$ & $1,52.10^{5}$ & 0 & 0 \\
\hline & V3 & 0 & 0 & 0 & 0 & - \\
\hline
\end{tabular}




\section{DISCUSSION}

La charge élevée de la FMAT favorise une forte altération du produit et constitue un risque de présence de germes pathogènes. La variabilité de la contamination en FMAT d'un vendeur à un autre d'une même localité pourrait dépendre de la densité de la fréquentation de la rue qui influe sur l'hygiène environnementale et donc de la pollution du produit.

La présence de Coliformes dans certains échantillons témoigne d'une hygiène défectueuse dans la transformation, pouvant découler du transformateur, du matériel en contact et/ou de l'environnement immédiat du produit. Ces bactéries ne sont généralement pas dangereuses du point de vue sanitaire sauf en cas de prolifération extrêmement abondante ou de sensibilité particulière du consommateur. On en tolère en général un nombre inférieur à $100 \mathrm{UFC/g}$ de produits. Ici, les échantillons V1 et V2 de Fann, V1 des HLM, V1 et V2 de Grand Yoff sont non conformes à ce critère microbiologique et sont par conséquent impropres à la consommation humaine.

La présence d'Escherichia coli dans certains échantillons (V1 et V2 des HLM, et V2 de Médina) atteste d'une contamination d'origine fécale, probablement humaine, et donc du transformateur. Ainsi, le niveau de contamination en Escherichia coli dans les échantillons V1 et V2 des HLM et V2 de Médina; et en entérobactéries dans les V1 et V2 de Grand Yoff et V3 des HLM, pourrait causer des risques sur la santé des consommateurs (norme ISO 16649-1 ou 2 cité dans le règlement $\mathrm{CE}$ 2073/2005). Conformément à cette norme, les limites de contamination en E. coli acceptables dans les fruits et légumes pré-coupés (prêt-à-manger) est comprise entre 100 et $1000 \mathrm{UFC} / \mathrm{g}$, ce qui n'est pas le cas pour ces échantillons.

La présence des quatre

entérobactéries (Enterobacter agglomerans, Enterobacter amnigenus Serrati rubidaea et Klebsiella pneumoniae) identifiées sur les galeries Api20E prouve, qu'en plus des coliformes, il y a des bactéries entériques dans les échantillons V2 de Médina, V1 de Fann et dans V1 et V2 de Guédiawaye. En effet, ces quatre (4) germes ne sont pas reconnus comme un danger sur la santé publique. Néanmoins, les échantillons concernés restent, en outre, non satisfaisants pour la consommation humaine au point de vue de leur qualité hygiénique.

Cependant, certains échantillons sont acceptables du point de vue de leur contamination en $E$. coli et entérobactéries. C'est le cas des échantillons V1, V2 et V3 de Fann, des échantillons V1 et V3 de la Médina et de l'échantillon V2 de Guédiawaye.

\section{Conclusion}

Au terme de cette étude, les résultats obtenus montrent une non satisfaction de tous les échantillons en flore totale à l'exception de l'échantillon V3 des HLM; mais aussi des conditions d'hygiène défectueuses marquées par une forte présence des coliformes et un niveau de contamination élevé en $E$. coli (> $10^{3} \mathrm{UFC} / \mathrm{g}$ ). D'autres entérobactéries comme Enterobacter agglomerans, Enterobacter amnigenus, Serrati rubidaea et Klebsiella pneumoniae ont été retrouvées respectivement dans les échantillons V2 de Médina, V1 de Fann, V1 et V2 de Guédiawaye. Cependant, certains échantillons restent acceptables pour la consommation humaine.

Ces premiers résultats mettent en évidence une nécessité d'élargir l'étude au niveau des différents quartiers de Dakar, avec un nombre plus élevé d'échantillons par quartier, afin de veiller sur la santé des consommateurs. Ceci participera en outre, à la mise en œuvre de mesures de surveillance de la qualité des mangues fraîches vendues en tranches dans des sachets plastiques au Sénégal.

Face à cette situation d'hygiène alimentaire défectueuse, de santé menacée des consommateurs, d'un désir nutritionnel ou d'une habitude alimentaire à satisfaire, d'une 
technologie à améliorer ou à valoriser et surtout d'emplois à sauver, des urgences s'imposent pour veiller à la qualité de nos produits alimentaires vendus dans les rues, mais aussi et surtout d'envisager des voies d'amélioration de leur conservation.

\section{REMERCIEMENTS}

Les auteurs remercient le Laboratoire de microbiologie appliquée et de génie industriel (MAGI) de l'ESP; le Programme d'Appui à l'Enseignement Supérieur de l'UEMOA (Union Economique et Monétaire Ouest Africaine) pour le financement de ce travail.

\section{REFERENCES}

ANSD (Agence Nationale de la Statistique et de la Démographique) du Sénégal. 2014. Carte administrative de la région de Dakar. ANSD.

Artès F. 2004. Le rôle du froid dans le maintien de la qualité et l'amélioration de la sécurité des produits alimentaires d'origine végétale. Bulletin de l'IIF $n^{\circ} 2004-1.14$ p. < En ligne >. http://www.researchgate.net/publication/2 42522537_Le_rle_du_froid_dans_le_main tien_de_la_qualit_et_l'amlioration_de_la_ scurit_des_produits_alimentaires_d'origin e_vgtale/file/9c96052d06d3cd4484.pdf, consulté le 18/11/2009.

Buffet F. 2003. Etude de la conservation de l'Ananas en produits prêt à consommer de $4^{\text {ème }}$ gamme. Rapport de stage, CIRADFHLOR de Montpellier, 48 p.

CIRAD (Centre de coopération Internationale de Recherches Agronomiques pour le Développement). 2009. Tout savoir sur les fruits et légumes des tropiques. Salon international de l'agriculture de Paris. <En ligne>. http://www.cirad.fr/ publications-ressources/science-pourtous/dossiers/fruits-legumes-destropiques/fruits-tropicaux/transformationdes-fruits, consulté le 16/09/2010

Cisse M, Vaillant F, Bouquet S, Pallet D,
Lutin F, Reynes M, Dornier M. 2011. Athermal concentration by osmotic evaporation of roselle extracts, apple and grape juices and impact on quality. Innovative Food Science and Emerging Technologies, 12: 352-360.

Commission regulation (EC) No 2073/2005. 2005. Microbiological criteria for foodstuffs. Official Journal of the European Union L338, 26p.

Djioua T. 2010. Amélioration de la conservation des mangues $4^{\text {ème }}$ gamme par application de traitements thermiques et utilisation d'une conservation sous atmosphère modifiée. Thèse de Doctorat en Sciences Agronomiques. Université d'Avignon et des Pays de Vaucluse, p. 150.

Djioua T, Charles F, Freire JM, Filgueiras H, Ducamp-Collin MN, Sallanon H. 2010. Combined effects of postharvest heat treatment and chitosan coating on quality of fresh-cut mangoes (Mangifera indica L.). International Journal of Food Science and Technology, 45: 849-855.

James IF, Kuipers B. 2003. La Conservation des Fruits et Légumes. Série Agridok N³ du CTA. Fondation «AGROMISA»: Wageningen, Pays Bas ; 94.

Kansci G, Koubala BB, Mbome LI. 2003. Effect of ripening on the composition and suitability for jam processing of different varieties of mango (Mangifera indica). African Journal of Biotechnology, 2(9): 301-306.

Kassé M. 2009. Évaluation des méthodes de contrôle phytosanitaire et qualité des produits horticoles importés et exportés au Sénégal. Mémoire Master II en Qualité des aliments de l'homme, Option Denrées d'origine végétale, EISMV de Dakar, p. 32.

Mbodj M. 2005. Le développement durable et la lutte contre la pauvreté: cas du Sénégal, la filière mangue d'exportation. Rapport final. Atelier ASPRODEB/SOM, p. 65. 
Reynes M, Ducamp MN. 2008. La valorisation des fruits tropicaux: Conservation et transformation des fruits à petite échelle. CIRAD. < En ligne >. http://www.cirad.fr/content/download/250 4/18484/version/1/file/Valorisation_fruits _tropicaux.pdf, consulté le 09/10/2009.

Sandhya. 2010. Modified Atmosphere Packaging of Fresh Produce: Current Status and Future Needs. LWT - Food Science and Technology, 43: 381-392.

Temple L. 2001. Quantification des productions et des échanges de fruits et légumes au Cameroun, Cahier d'Agriculture, 119(10): 87-94.
Thuan BH. 2003. Conservation en frais des fruits et légumes. Cours, Université de Cantho, 22 Novembre 2003, Vietnam. AUF. < En ligne >. http://www.Infotheque.info/ressource/611 6.html, consulté le 02/01/2009 et http://www.vn.refer.org/mp/ctu_fruits/fra me.htm, consulté le 17/10/2010.

Varoquaux P. 2002. Fruits frais prêts à l'emploi dits de $4^{\text {ème }}$ gamme. In Technologies de Transformation des Fruits. Albagnac (ed). Lavoisier : Paris; 119-156. 